\title{
The MOST and COROT prime target fields: A target inventory
}

\author{
J. Oehlinger, A. Kaiser, T. Kallinger, P. Mittermayer, W. Weiss, K. Zwintz \\ Institut für Astronomie, Türkenschanzstrasse 17, 1180 Vienna, Austria
}

\begin{abstract}
MOST and COROT are two satellite missions dedicated to asteroseismology and the detection of exoplanets. Both satellites use CCDs as a detector and hence they do not exclusively observe their prime targets but an entire field with additional objects. It is crucial to know their astrophysical properties and to have information on the location in the CCD-fields. Therefore VISAT (Vlenna Selection of Astronomical Targets, see Kallinger et al., this volume) was used to investigate the fields around the prime targets of COROT and MOST resulting in the two catalogues described in this article.
\end{abstract}

\section{Introduction}

MOST (Microvariability and Oscillation of STars) is a Canadian microsatellite performing asteroseimology of sun-like and magnetic stars as well as studying microvariability in Wolf-Rayet winds and other targets. It is a low budget mission which demonstrates that even small missions can perform high quality observations. MOST has been launched by Eurockot on June 30th, 2003, from Plesetsk, Russia, with a Proton rocket and operates at an altitude of $820 \mathrm{~km}$ in a polar dawn-dusk orbit. The optical system consists of a 15-cm-Maksutov telescope and a CCD camera (1024 by 1024 pixels). An array of 36 Fabry lenses in the focus of the telescope (Fig. 1) minimizes the effect of satellite jitter and provides the required photometric precision (Matthews 1998).

COROT (COnvection, ROtation and planetary Transits) is a French-lead satellite mission with the cooporation of several, mainly European countries which is dedicated to asteroseismology and the discovery and study of exoplanets with the transit technique. Launch date is mid 2006 and COROT will perform its observations from an $800-900 \mathrm{~km}$ polar orbit. COROT is equipped 


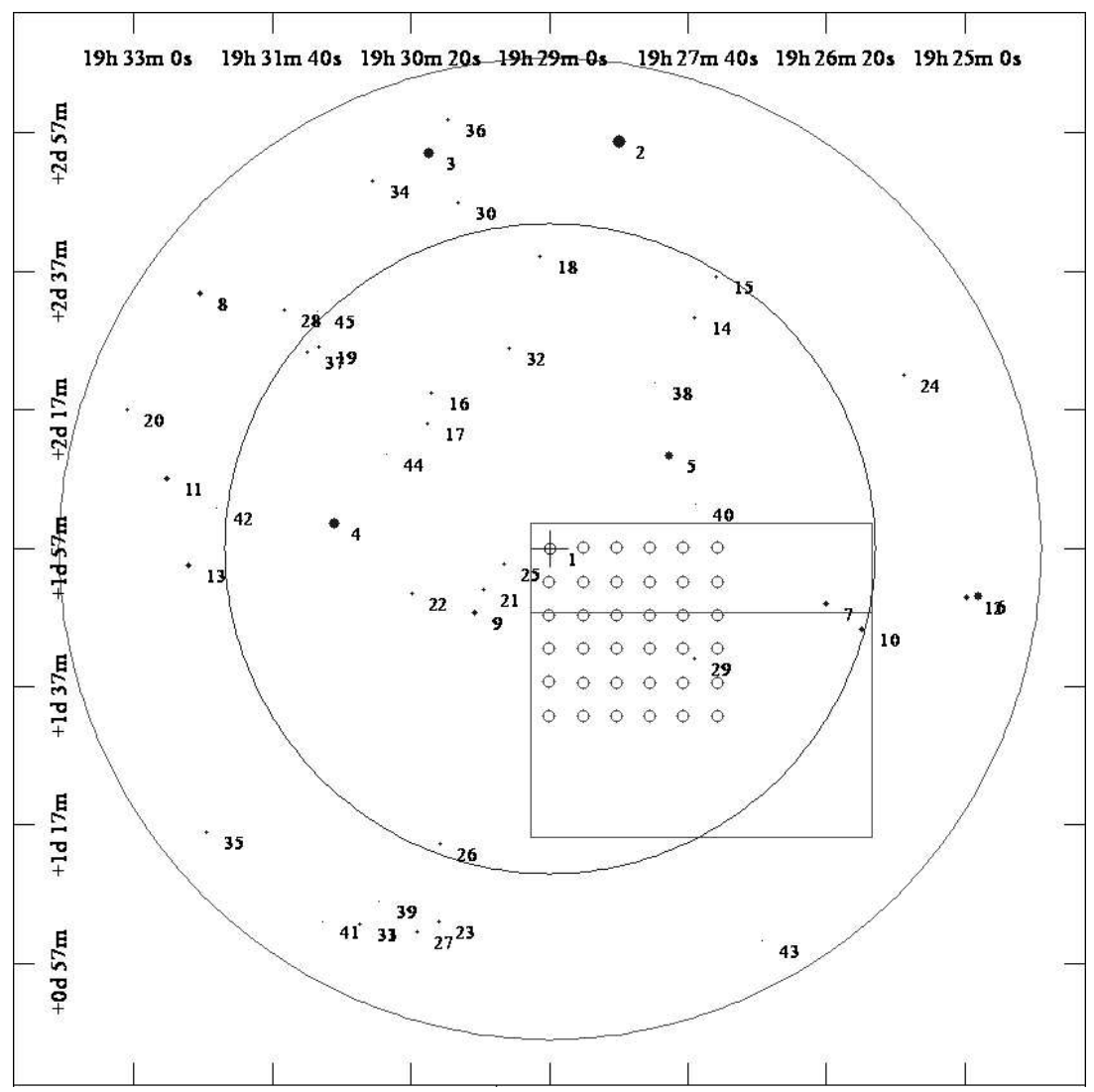

Figure 1: MOST chart of HD 183324 as produced by VISAT. The smaller circle represents the accessible area for the MOST camera for HD 183324. The target star can be positioned in one of the 36 fabry lenses and the CCD can be rotated at an angle of 360 degrees. In inserted rectangular illustrates the CCD and the location of the Fabry lenses.

with a $27 \mathrm{~cm}$ telescope and two CCDs (1024 by 1024 pixels) for asteroseismology and exoplanet search each (Baglin et al. 2002). The satellite can be rotated around the optical axis by an angle of \pm 20 degrees (see Fig. 2).

Many interesting objects can be found in the field of view (FOV) of those satellites, as is illustrated in Fig. 3. The orientation of the CCD's projected on the sky defines the observable targets and hence the amount of accessible science. To optimize the scientific output, information about the astrophysical properties is required and also their location in the FOV. 


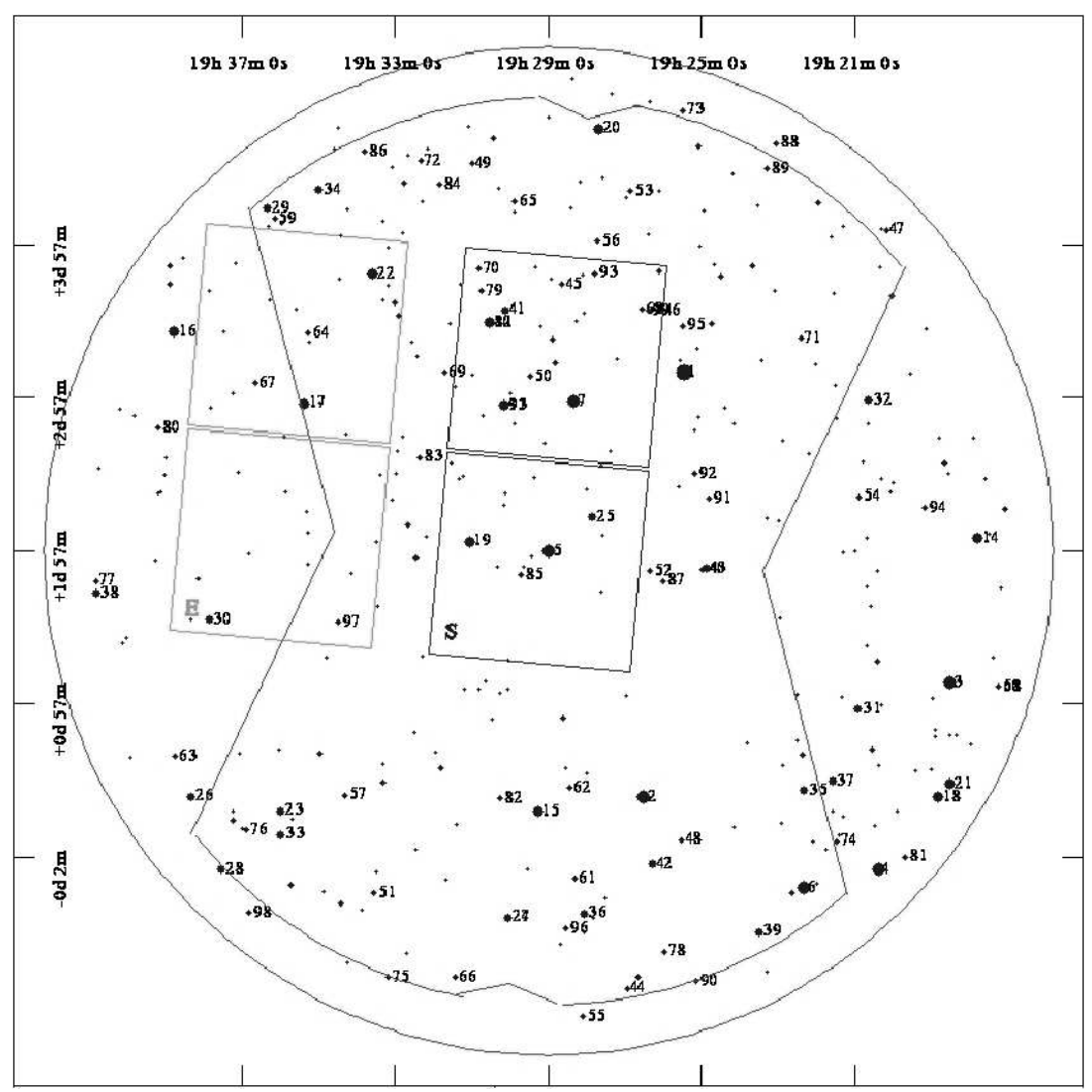

Figure 2: COROT star chart for HD 183324. The two rectangles represent the CCD's for the asteroseismology (left) and the exoplanet program (right). The dumbellshaped figure represents the accessible area for the COROT seismology CCD's when HD 183324 is anywhere on the seismology field. The field of view can be rotated by an angle of \pm 20 degrees.

The present investigation is an attempt to characterize the area around the prime targets and to provide this information to the scientific community for preparing additional science programs. For this reason we analyzed the fields using the VISAT database, which contains catalogs and information, based also on private communications, specialized in different types and objects, such as $\delta$ Scuti stars, rapidly oscillating Ap (roAp) stars, etc. (see T. Kallinger et al., this volume). 


\begin{tabular}{|c|c|c|}
\hline \multicolumn{2}{|c|}{ MOST targets } & COROT targets \\
\hline HD 61421(Procyon) & HD 38529 & HD 171834 \\
\hline HD 121370 ( $\eta$ Boo $)$ & HD 191765 (WR 134) & HD 174866 \\
\hline HD 10700 ( $\tau$ Cet $)$ & HD 192103 (WR 135) & HD 183324 \\
\hline HD $102870(\beta$ Vir $)$ & HD 17723 (WR 123) & HD 184663 \\
\hline HD 142860 ( $\gamma$ Ser $)$ & 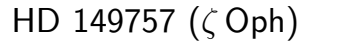 & HD 43318 \\
\hline HD 99028 ( $\iota$ Leo $)$ & HD 114710 ( $\beta$ Com $)$ & HD 43587 \\
\hline HD 224930 & HD $22049(\epsilon$ Eri) & HD 46304 \\
\hline HD 76932 & HD 120136 ( $\tau$ Boo $)$ & HD 45067 \\
\hline HD $201601(\gamma \mathrm{Equ})$ & HD 209458 & HD 46304 \\
\hline HD 176232 (10 Aql) & HD 165688 (WR 110) & HD 49933 \\
\hline HD 24712 (HR 1217) & QR Sge (WR 124) & HD 55057 \\
\hline HD 217014 (51 Peg) & HD 56925 (WR 007) & HD 57006 \\
\hline
\end{tabular}

Table 1: The MOST and COROT prime targets, analyzed in this paper

\section{Target inventory}

The catalogs of the MOST and COROT field analyses contain a VISAT star chart and an object list for each prime target, which contains any entry found in the database. A list of the analyzed MOST and COROT prime target stars can be found in Tab. 1.

Fig. 1 and Fig. 2 show the star charts of the MOST and COROT mask produced by VISAT. The circle in Fig. 1 and the dumbell-shaped figure in Fig. 2 represent the accessible area for MOST and COROT, provided that the prime target is anywhere in the photometric area of the CCD. All objects shown in this charts are stars which have entries in the VISAT database.

Fig. 3 shows an example of an object list. As a star can be found in different catalogues, multiple entries are possible. Among star identifier and coordinates, the list contains also information on the spectral type and photometric parameters extracted from SIMBAD. As the catalogs implemented in VISAT provide different spectral classifications, multiple spectral types are possible. If a star was only found in one catalogue, the spectral type entry from SIMBAD was added. Absolute lumniosity $\left(M_{V}\right)$ was either calculated using Hipparcos parallaxes or using the calibration given by Gray (1992).

Furthermore, HR-diagrams of the COROT fields were generated (Fig. 4). Full circles represent the datapoints with $\mathrm{M}_{V}$, calculated with Hipparcos parallaxes, the light stars were obtained using the calibration given by Gray. A few regions for variable stars, like the instability strip and the regions for $\beta$ Cephei, SBP and $\gamma$ Dor stars were added in the HR-diagrams.

The complete MOST catalogues for all the prime targets presently chosen 


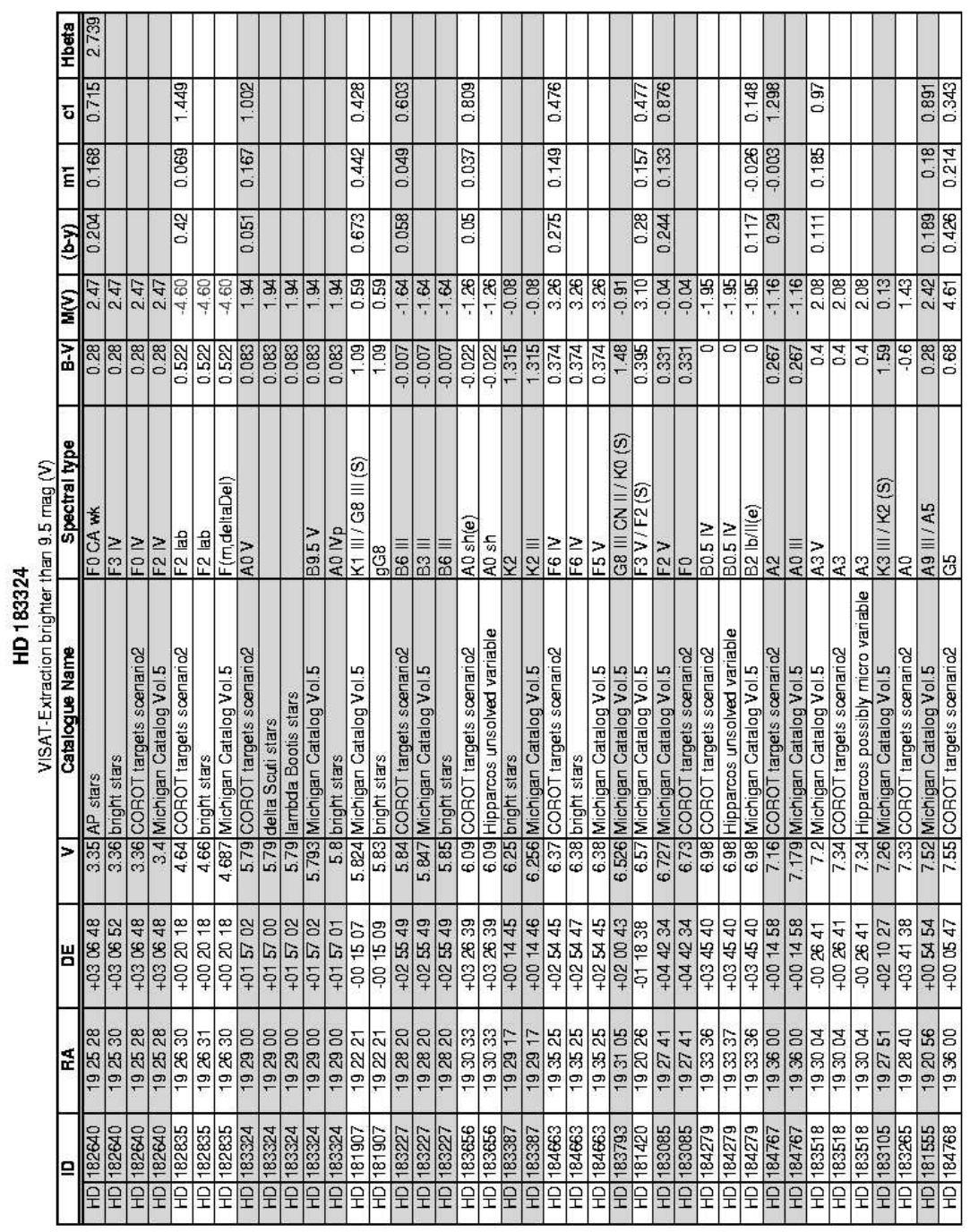

Figure 3: Object list of the field stars around HD 183324. As one star can be found in different catalogues implemented in VISAT, multiple entries are possible. If there was only one spectral type entry found, a SIMBAD entry was added which was marked with (S). The photometric parameters were extracted from SIMBAD, $\mathrm{M}_{V}$ was calculated using Hipparcos data (SIMBAD) or the calibration from Gray, F. D. 
HD 183324

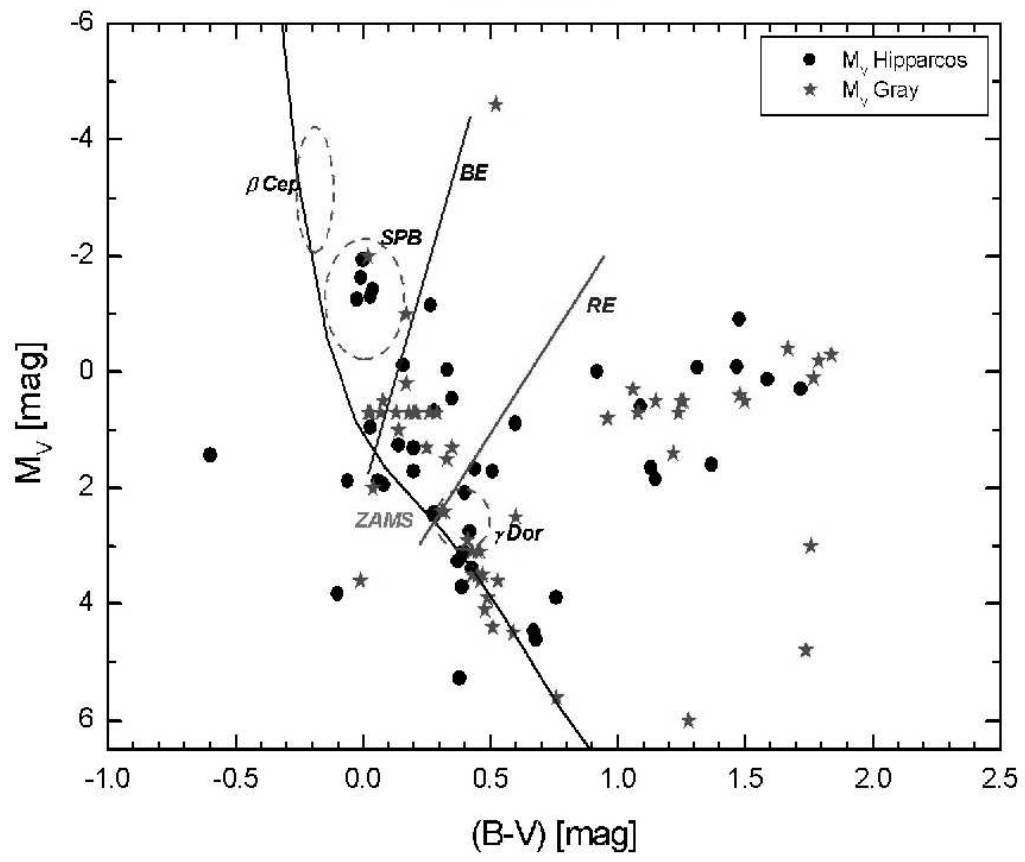

Figure 4: HR-diagram of the field around HD 183324. Full circles represent data calculated using Hipparcos data (extracted from SIMBAD). Lighter stars represent data which were calculated using the calibration given by Gray, F. D. The blue and red edge of the instability strip as well as some further regions where variable stars can be found are added.

for the first two years of operation can be downloaded from

http://ams.astro.univie.ac.at/space/mostinventory.pdf and for COROT from

http://ams.astro.univie.ac.at/space/corotinventory.pdf (COROT).

Acknowledgments. This research made use of the SIMBAD database, operated at CDS, Strasbourg, France. Financial support was received from the Bundesministerium für Verkehr, Innovation und Technologie and the Austrian Space Agency (MOST), the Bundesministerium für Bildung, Wissenschaft und Kultur (COROT) and the Austrian Fonds zur Förderung der wissenschaftlichen Forschung (project P14984). 


\section{References}

Matthews, J. 1998, Cassiopeia, CASC newsletter, No. 96

Baglin, A., Auvergne, M., Barge, P., Buey, J.-T., Catala, C., Michel, E., Weiss, W. W., and the COROT Team 2002, COROT: asteroseismology and planet finding,Proceedings of the First Eddington Workshop, ESA SP-485, p. 17

Gray, F. D. 1992, The observation and analysis of stellar photospheres, Second Edition, (Cambridge, Cambridge University Press), p.430-434

Kallinger, T., Zwintz, K., Kaiser, A., Mittermayer, P., Weiss, W. W., 2003, CoAst, Vol. 143 